\title{
Non-intrusive aerodynamic loads analysis of an aircraft propeller blade
}

\author{
D. Ragni • B. W. van Oudheusden • \\ F. Scarano
}

Received: 19 November 2010/Revised: 27 January 2011/ Accepted: 4 February 2011/Published online: 22 February 2011

(C) The Author(s) 2011. This article is published with open access at Springerlink.com

\begin{abstract}
The flow field in a cross-sectional plane of a scaled Beaver DHC aircraft propeller has been measured by means of a stereoscopic PIV setup. Phase-locked measurements are obtained in a rotational frequency range from 18,900 to $21,000 \mathrm{rpm}$, at a relative Mach number of 0.6 at $3 / 4$ propeller radius. The use of an adapted formulation of the momentum equation in differential form for rotating frame of references, integrated with isentropic relations as boundary conditions, allowed to compute the pressure field around the blade and the surface pressure distribution directly from the velocity data in the compressible regime. The procedure, extended to the computation of the aerodynamic lift and drag coefficients by a momentum contour integral approach, proved to be able to couple the aerodynamical loads to the flow field on the moving propeller blade, comparing favorably with a numerical simulation of the entire scaled model. Results are presented for two propeller rotation speeds and three different yawing angles.
\end{abstract}

\section{Introduction}

Propeller engines as propulsion devices have wide application in aeronautics for their favorable propulsive efficiency, leading to lower fuel consumption and longer endurance in comparison with turbofans and turbojets. However, the complex design of modern aircraft propellers, together with the high revolution frequency, limits the availability of experimental results to the slipstream

D. Ragni $(\bowtie) \cdot$ B. W. van Oudheusden · F. Scarano

Faculty of Aerospace Engineering,

Delft University of Technology, Delft, The Netherlands

e-mail: D.Ragni@tudelft.nl development, to the validation of Euler algorithms (Roosenboom et al. 2010; Boyle et al. 1999), or to the investigation of wakes in free-axial flight (Favier et al. 1989; Ramasamy and Leishman 2006). In contrast, experimental data in the field of naval engineering are more widely available (Calgano et al. 2005), where the focus ranges from the interaction effects between the propeller wake and the ship hull, to the fatigue and corrosion caused by cavitation at high revolving frequencies. Despite the advances of computational fluid dynamics, which broadened the range of investigation to the unsteady high-speed propellers aerodynamics, the co-occurrence of high Mach numbers with unsteady flow still affects the accuracy in the computation of the flow field (Bousquet and Gardarein 2003).

In this respect, the use of non-intrusive techniques as particle image velocimetry PIV or laser Doppler anemometry LDA becomes fundamental in understanding and validating the numerical computations, providing information about the local flow velocity and accelerations, moreover, whenever retrieving simultaneous information about the flow velocity and the forces acting locally on the blade profile, in the presence of separation and shock waves. The determination of aerodynamic loads on airfoils by means of PIV, using a momentum-balance approach, has already been proven to be a valid non-intrusive method; in particular, in the low-speed regime, van Oudheusden et al. (2006) demonstrated how aerodynamic loads can be extracted from PIV 2D/2C fields on a NACA642A015 airfoil. In the transonic regime, Ragni et al. (2009) similarly showed that the computation of airfoil drag and lift coefficients can be obtained with a reasonable agreement with well-proven conventional techniques.

In the present investigation, such an approach is extended to the periodical unsteady rotational motion of a scaled model of a DHC Beaver aircraft propeller, running in the 
compressible regime, by use of a modified version of the momentum and energy equation in the relative frame of reference, able to provide information about the static and relative total pressure and temperature, about the pressure field around the blade profile, and about the loads in the blade profile plane. The technique, able to couple the flow velocity with the action-reaction on the blade itself, shows potentials in combining the global optimization of the aerodynamical propeller performances, with a more detailed local profile shape analysis. Phase-locked velocity measurements are presented for two revolution frequencies and three yawing angles, together with the planar pressure reconstruction and the computation of the local sectional aerodynamic coefficients. Due to the impracticability of equipping the blade with pressure orifices, a numerical periodically steady Reynolds averaged Navier-Stokes (RANS) computation was employed, in particular for the fastest regime $(21,000 \mathrm{rpm})$ analyzed.

\section{Experimental procedures}

\subsection{Wind-tunnel, model, and operating regimes}

The PIV experiments on the propeller flow were performed in the low-speed, closed-circuit wind-tunnel (LST) of the Low Speed Aerodynamics Laboratories at the Delft University of Technology. The LST facility has a cross-section

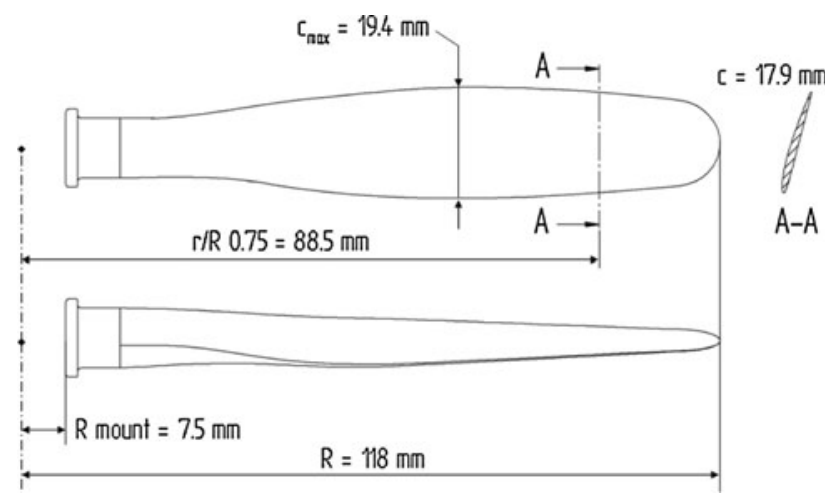

Fig. 1 Propeller blade geometry of $1.8 \mathrm{~m}$ width and $1.2 \mathrm{~m}$ height and operates up to $120 \mathrm{~m} / \mathrm{s}$ at ambient pressure $(101.3 \mathrm{kPa})$. The 4-bladed scaled model of a DHC Beaver aircraft propeller of $236 \mathrm{~mm}$ diameter was installed in the center of the test section. The propeller is driven by a $5.6 \mathrm{~kW}(7.5 \mathrm{hp})$ electrical engine, displaced by a supporting sting, providing cooling to the system by means of an internal water circuit. A balance actuator enables the movement of the yawing angle of the propeller, in a range from -10 to 10 degrees, while an embedded angular position encoder allowed synchronizing the PIV measurements with the blade position to obtain phase-locked measurements. Figure 1 shows the main dimensions of the blade together with the profile section at $3 / 4$ of the blade radius.

The two fastest revolution frequencies investigated, keeping the engine temperature below $120^{\circ} \mathrm{C}$, are $330 \mathrm{~Hz}$ and $350 \mathrm{~Hz}$ corresponding to 19,800 and $21,000 \mathrm{rpm}$, respectively. The two regimes together with a wind-tunnel free-stream velocity of $47.8 \mathrm{~m} / \mathrm{s}$ allowed having transonic conditions at $3 / 4$ propeller radius, with a maximum propeller slip (ratio between the free-stream velocity and the advance propeller velocity) $s=1-V_{\infty} / V_{p}$ of $5 \%$. Details on the propeller characteristics and on the operating regimes are presented in Table 1.

\subsection{PIV measurement apparatus}

A stereoscopic PIV experiment was conducted to measure the flow over several cross-sections of the airfoil. The flow is seeded with particles produced by a SAFEX Twin Fog generator with SAFEX-Inside-Nebelfluid, a mixture of diethylene glycol and water creating seeding droplets of 1 micron median diameter. The particles are introduced in the LST wind-tunnel, directly downstream of the test section, and are uniformly mixed during the recirculation. The model is illuminated by a Quantel CFR200 Nd-Yag laser with $200 \mathrm{~mJ} /$ pulse energy. Light sheet optics produce a laser sheet of $2 \mathrm{~mm}$ thickness ( $30 \mathrm{~cm}$ wide). Two LaVision Imager Pro LX cameras with $4,872 \times 3,248$ pixels (10 bit) and two Nikon lenses of $105 \mathrm{~mm}$ focal length at $f \# 8$ have been used with the LaVision Davis 7.2 software for acquisition and post-processing. Sets of 150 images have
Table 1 Blade geometry and summary of propeller characteristics with operating conditions

\begin{tabular}{llll}
\hline Propeller geometry & \multicolumn{3}{c}{ Operating regimes } \\
\hline Number of blades $N$ & 4 & Blade revolution frequency & $330-350 \mathrm{~Hz}$ \\
Propeller radius $R$ & $118 \mathrm{~mm}$ & Rotational velocity at $r / R=0.75$ & $189.1 \mathrm{~m} / \mathrm{s}$ \\
Assembling tolerance & $7.5 \mathrm{~mm}$ & Advance free-stream velocity & $47.8 \mathrm{~m} / \mathrm{s}$ \\
Blade maximum chord c & $19.4 \mathrm{~mm}$ & Relative Mach at $r / R=0.75$ & 0.63 \\
Blade chord at $r / R=0.75$ & $17.9 \mathrm{~mm}$ & Chord Reynolds number & 310,000 \\
Pitch angle at $r / R=0.75$ & $15 \mathrm{deg}$ & Advance ratio $J$ & $0.58-0.62$ \\
Solidity ratio $\mathrm{c} N /(\pi R)$ & 0.21 & Real pitch/Ideal pitch & 0.92 \\
\hline
\end{tabular}


Fig. 2 Stereoscopic PIV setup and details of the apparatus

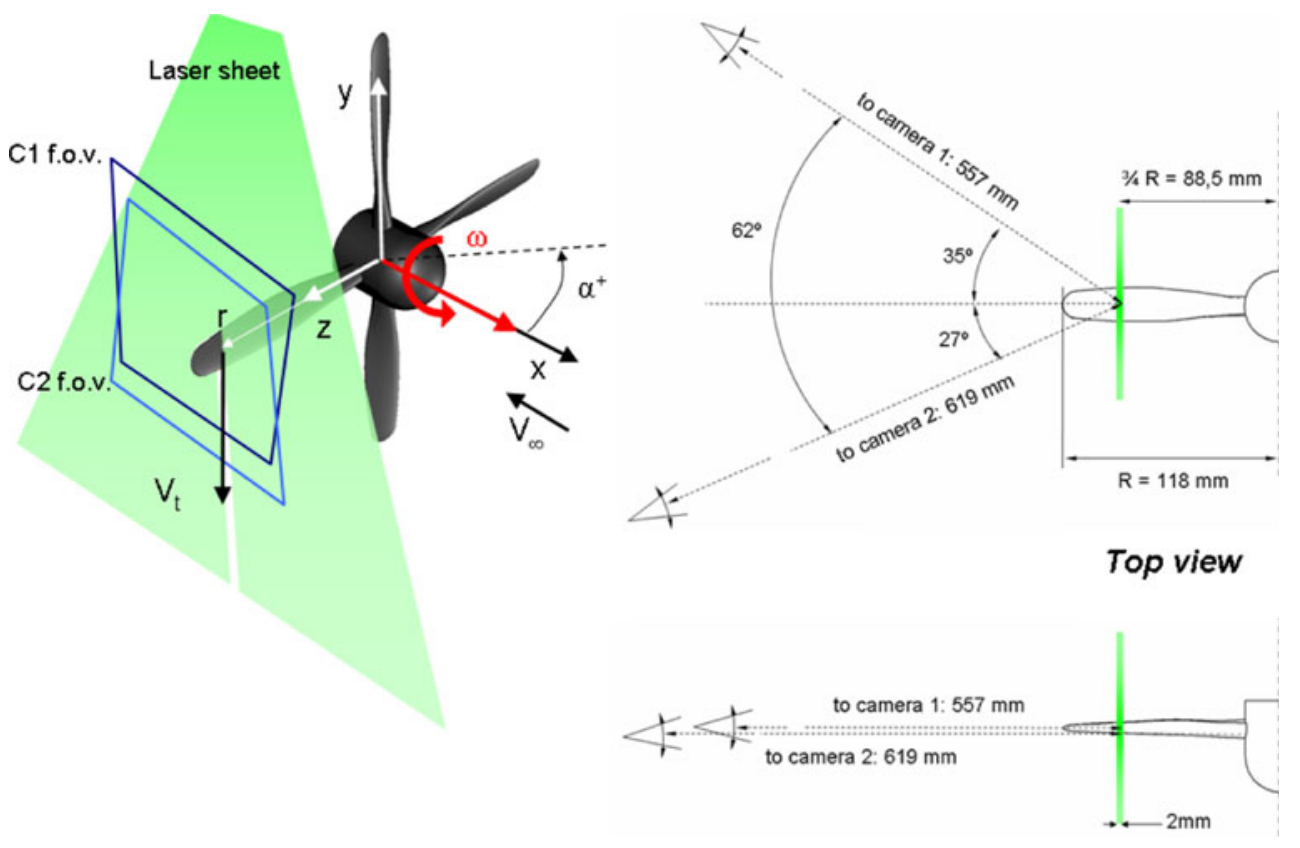

been recorded in phase-locked mode at a maximum frequency of $2.5 \mathrm{~Hz}$. The recordings are evaluated with a window deformation iterative multi-grid (Scarano and Riethmuller 2000) with window size from $16 \times 16$ pixels $50 \%$ overlap down to $4 \times 4$ pixels $0 \%$ overlap (5 vectors per $\mathrm{mm}$ ), and subsequently averaged. In Fig. 2, a schematic of the setup is presented together with summary of the PIV parameters in Table 2.

Two engine-embedded encoders were used to control the propeller revolution frequency and to synchronize the acquisition system, phase-locking the measurements on the laser plane, perpendicular to the propeller blade axis. The first control unit could maintain the revolution frequency constant within $\pm 0.3 \mathrm{~Hz}$ (less than $0.1 \%$ at $350 \mathrm{~Hz}$ ), while the second one provided the acquisition phase with an uncertainty corresponding to a jitter of the blade position on the raw image of less than $1 \mathrm{px}(50 \mu \mathrm{m})$.

In order to compute the pressure field and the aerodynamic loads, the governing equations require determination of the spatial in-plane and out-of-plane derivatives, as well as the time derivatives of the velocity fields (see Sect. 4). Information on the time development of the flow is obtained by delaying the system from a known reference encoder signal with a Stanford control unit, allowing to image the propeller blade at different time instants, in particular at $\pm 5, \pm 10, \pm 20 \mu$ s from the reference, the uncertainty of which has been found to be negligible compared to the raw image scatter. The spatial derivatives in out-of-plane direction were obtained from subsequent measurement of multiple planes displaced in the span-wise direction of the blade. The span-wise position was calibrated by placing the laser system on a micrometer bench,
Table 2 Stereoscopic PIV setup and details of the apparatus

\begin{tabular}{lc}
\hline Illumination & \\
\hline Laser & Quantel CFR 200 Nd:Yag \\
Pulse energy & $200 \mathrm{~mJ}$ \\
Light sheet thickness & $2 \mathrm{~mm}$ \\
\hline Imaging & 2 Imager Pro LX \\
\hline Camera & $4,872 \times 3,248 \mathrm{px}$ \\
Sensor format & $7.40 \mu \mathrm{m}$ \\
Pixel pitch & $105 \mathrm{~mm}$ \\
Lenses focal length & 0.148 \\
M & $19-23 \mathrm{px} / \mathrm{mm}$ \\
Imaging resolution & $\sim 18 \times 12 \mathrm{~cm}{ }^{2}$ \\
FOV & \multicolumn{1}{c}{ From 0.8 to $0.2 \mathrm{~mm}$} \\
Spatial resolution & \\
\hline Acquisition & LaVision Davis 7.2 \\
\hline Software & $1.5-2.5 \mathrm{~Hz}$ \\
Frequency & $15 \mu \mathrm{s}$ \\
Pulse separation & $150-200$ \\
Number of recordings & From $16 \times 16$ to $4 \times 4 \mathrm{px}$ \\
Window size &
\end{tabular}

with an overall accuracy of a tenth of a $\mathrm{mm}$ relative to a $\pm 2 \mathrm{~mm}$ laser sheet overall movement.

\subsection{Computational fluid dynamics model}

To obtain comparison data for the experimental study, a numerical simulation was made of the flow around the 


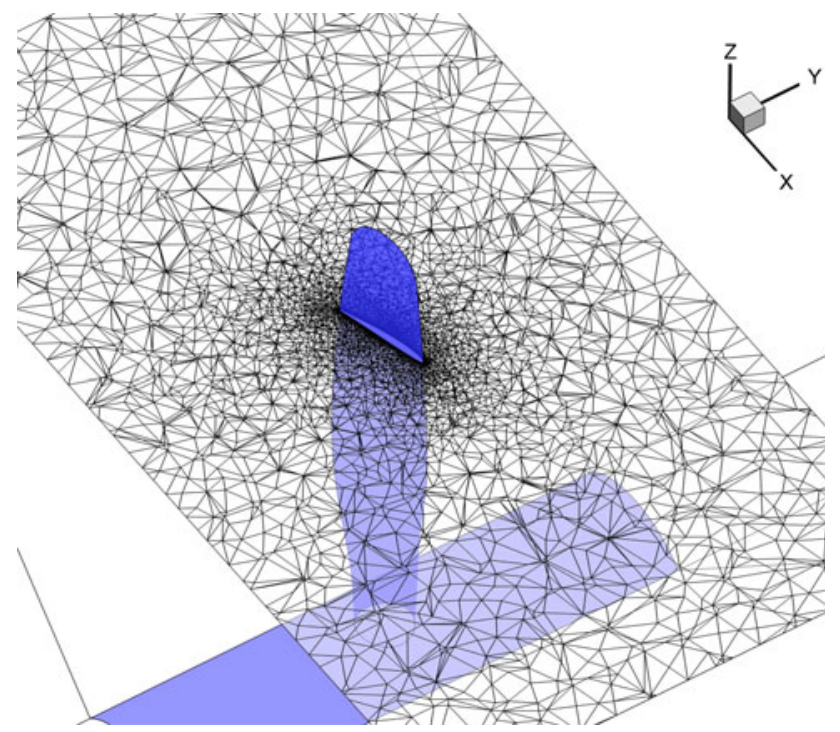

Fig. 3 Slice detail of the periodical CFD propeller model

aircraft propeller in the zero-yaw case. The geometry of the blade has been replicated on a CAD Solid Edge model and imported in the commercial CFD simulation program Fluent V.12.1, where the experimental fastest regime has been simulated by use of a 3D steady-periodical RANS model, consisting of a $90^{\circ}$ sector meshed with three volumes, with the one of the blade on a moving frame of reference,. The periodical implementation helps the modeling by reducing the number of nodes needed, since just one of the propeller blades has to be meshed. Mapped elements on the surrounding volumes and tetrahedral in the moving one were used; the final mesh ensured a $y^{+}$value of $1\left(y_{0}\right.$ at $\left.1.3 \times 10^{-3} \mathrm{~mm}\right)$ in the direction normal to the blade surface with a maximum of 2,069,574 nodes. A oneequation Spalart-Allmaras turbulence model has been used with Standard Wall Functions and a third-order model for the flow discretization. A subsequent structured CFD computation of the entire propeller with more than 30 millions nodes and a normal surface $y^{+}$of $0.8\left(y_{0}\right.$ at $1.1 \times 10^{-3} \mathrm{~mm}$ ) on a single rotating volume was used only to validate the results of the periodical mesh used as a PIV comparison (Fig. 3).

\section{Data reduction}

\subsection{Frames of reference}

The properties of the flow around the propeller blade can be evaluated in either a stationary or a moving frame of reference. The first formulation defines the velocity vectors as measured with respect to an inertial observer seeing the propeller revolving at angular velocity $\omega$. The moving- frame formulation, on the other hand, defines the velocity vectors as measured by a non-inertial observer moving together with the blade at the same angular velocity. If the blade is imaged perpendicularly to its axis, and the dimension of the analyzed field is relatively small compared to the propeller radius, the rotational component can be approximated by a translational motion.

In Fig. 4, the situation obtained in the two frames is depicted using both a conceptual drawing and an exemplary velocity field from the acquired measurement data. The two drawings on the left side of Fig. 4 graphically show how the combination of the wind-tunnel free-stream $\bar{V}_{\infty}$ and the blade velocity $\bar{\omega} \times \bar{r}$ is generating the two vector fields on the right side, in the stationary frame (topright) and in the relative frame (bottom-right), respectively. Both the drawing and the data in Fig. 4-top from the stationary frame of reference highlight the fact that the stagnation point is formed on the suction side of the blade profile, due to the free-stream flow coming from the top. When plotted in the moving frame, with the rotational component of the blade motion subtracted, the main features of a cambered airfoil flow are recovered; being the flow accelerating on the suction and partly on the pressure side.

The static fluid-dynamic quantities are independent on the flow velocity and therefore, are invariants for the two frames of reference. Instead, due to the added energy of the moving object, the total flow properties, such as the total pressure, temperature, density $P_{0}, T_{0}, \rho_{0}$, have different values in the two frames. Assuming that no thermal energy is added, the flow can be considered isentropic in the rotating reference frame moving with the propeller, with the total quantities given by:

$$
\begin{aligned}
T_{R 0} & =T_{\infty}+\frac{1}{2 c_{p}}\left|\bar{V}_{R \infty}\right|^{2} \\
P_{R 0} & =P_{\infty}\left(1+\frac{\gamma-1}{2} M_{R \infty}^{2}\right)^{\frac{\gamma}{\gamma-1}} \quad M_{R \infty}=\frac{V_{R \infty}}{\sqrt{\gamma R T_{\infty}}}
\end{aligned}
$$

where $c_{p}$ is the specific heat, $M$ the Mach number, $R$ the ideal gas constant, $\gamma$ the adiabatic index; the subscript ${ }_{R}$ stands for relative (moving) frame, and the relative velocity is $\bar{V}_{R}=\bar{V}-\bar{\omega} \times \bar{r}$, where $\omega$ is the angular velocity and $\bar{r}$ is the position vector. These relations express how the motion energy of the blade is transferred to the flow in the form of total pressure and temperature increase.

\subsection{Pressure computation}

In absence of large unsteady effects, the flow field can be assumed to be phase steady in the moving frame of reference. Therefore, in the region where the flow behaves as adiabatic and inviscid, the isentropic relations (Anderson 
Fig. 4 Velocity field representation, top stationary frame formulation, bottom moving-frame formulation
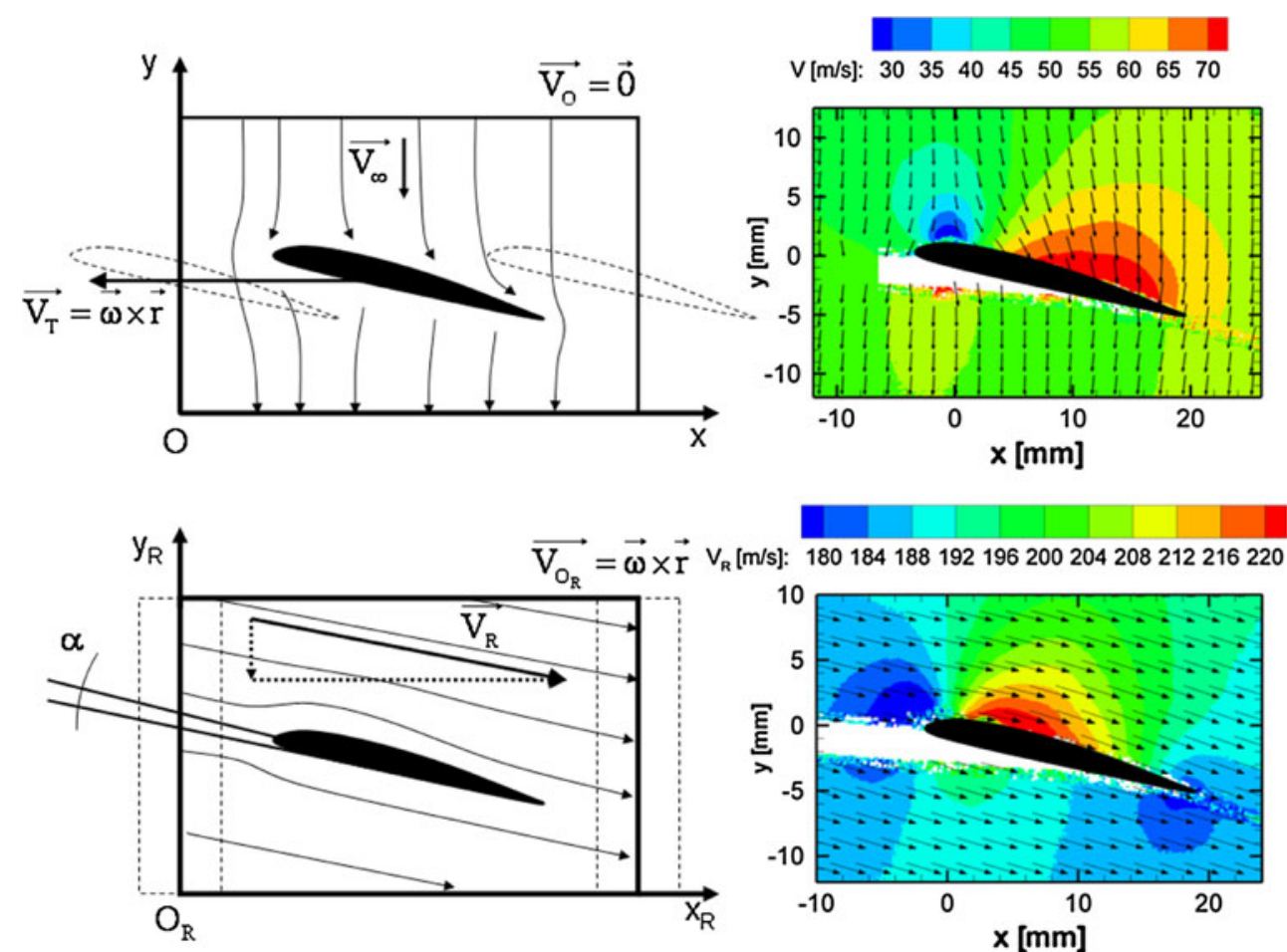

s]: 180184188192196200204208212216220

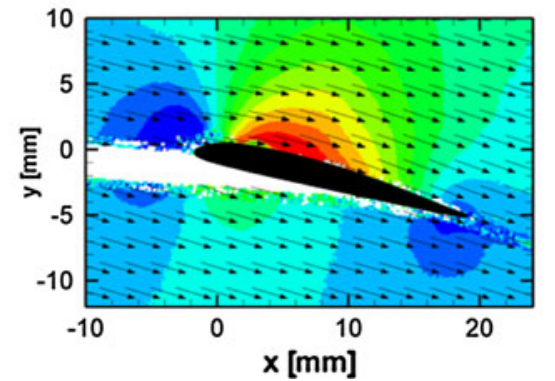

1991) can be used to compute the pressure directly from the local velocity:

$\frac{P}{P_{\infty}}=\left[1+\frac{(\gamma-1)}{2} M_{R \infty}^{2}\left(1-\frac{V_{R}^{2}}{V_{R \infty}^{2}}\right)\right]^{\gamma /(\gamma-1)}$

with $V_{R}=\left|\bar{V}_{R}\right|$ being the relative velocity magnitude. The previous equation allows writing the isentropic estimate of the pressure coefficient $C_{p}$ only depending on the relative Mach number:

$C_{p}=\frac{P-P_{\infty}}{\frac{1}{2} \rho_{\infty} V_{R \infty}^{2}}=\frac{2}{\gamma M_{R \infty}^{2}}\left(\frac{P}{P_{\infty}}-1\right)$

In rotational flow regions, for the major part represented by the wake of the blade, a different strategy needs to be applied for the pressure field determination using the momentum equation in differential form (Ragni et al. 2009). In rotational regions where the viscous terms represent a negligible contribution in the momentum equation, the Euler equations can be used. The pressure distribution is therefore computed from a spatial integration of the differential form of the momentum equation by a $2 \mathrm{D}$ Poisson second-order accurate scheme implemented on the basis of Trefethen (2000), imposing the isentropic pressure in the free stream. Assuming adiabatic flow, the static temperature differences solely depend upon the local velocity, while perfect gas behavior allows the variable density to be eliminated, providing a differential relation between velocity data and pressure (van Oudheusden et al. 2007): $\frac{\bar{\nabla} P}{P}=\bar{\nabla} \ln \left(P / P_{\infty}\right)=-\frac{1}{R T} \frac{D \bar{V}}{D t}$

The previous formulation can be simplified in the moving frame, by neglecting the unsteady components and by substituting the stationary velocities with the relative ones.

$$
\begin{aligned}
\bar{\nabla} \ln \left(P / P_{\infty}\right) & =-\frac{1}{R T}\left(\bar{V}_{R} \cdot \bar{\nabla}\right) \bar{V}_{R} \\
& =-\frac{\gamma M_{R \infty}^{2}}{V_{R \infty}^{2}+\frac{\gamma-1}{2} M_{R \infty}^{2}\left(V_{R \infty}^{2}-V_{R}^{2}\right)}\left(\bar{V}_{R} \cdot \bar{\nabla}\right) \bar{V}_{R}
\end{aligned}
$$

Reynolds turbulent stresses were found to have a negligible effect in earlier transonic airfoil studies (van Oudheusden et al. 2006; Ragni et al. 2009) and, consequently, have not been included in the current pressure evaluation. More extensive details of the pressure gradient evaluation in compressible flows, including the effect of the turbulent stresses, can be found in van Oudheusden (2008).

\subsection{Force determination by momentum integral}

The force acting on a body immersed in a fluid is the result of the surface pressure and shear stress distributions. Application of the integral momentum conservation concept permits the integral forces acting on the body to be computed from their reaction on the flow, without the need to explicitly evaluate the flow quantities at the surface of 


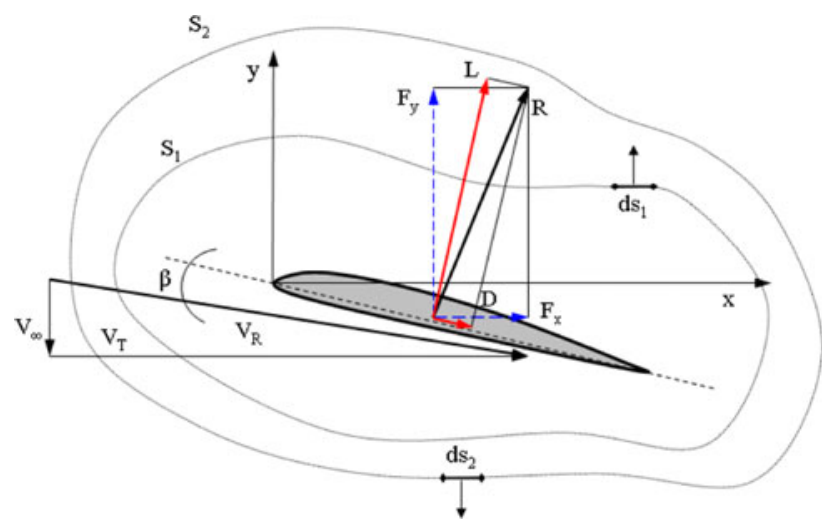

Fig. 5 Schematic of the integral momentum approach

the model (Anderson 1991). A schematic of the approach is depicted in Fig. 5.

In the stationary frame of reference, the momentum equation in its integral form relates the resultant aerodynamic force $\bar{R}$ on the airfoil to a contour integral around it (Unal et al. 1997):

$\bar{R}=-\frac{d}{d t} \iiint_{V} \rho \bar{V} d v-\oint_{S} \rho[\bar{V} \cdot d s \bar{V}]-\oint_{S}(p \overline{\bar{I}}-\overline{\bar{\tau}}) \cdot \overline{d s}$

In the above formulation, $S$ is the outer contour of an arbitrary volume $V$ containing the airfoil, on which the pressure $p$ and stress contribution $\tau$ act, the latter incorporating both viscous and turbulence effects. As previously mentioned, viscous and turbulent stresses along the contour are neglected henceforth, as they do not play a significant role when the integration contour is at a sufficient distance from the body.

In the moving-frame formulation the relative velocities are considered, while according to the quasi-steady assumption, the time derivative is omitted, so that no volume integration needs to be performed. Note that in the present approach, the velocity fields are obtained by phaseaveraged measurements; therefore, the time derivative applies to the periodic variation imposed by the propeller revolution.

The resultant aerodynamic force may be resolved into the Cartesian $x, y$ components or into lift and drag with respect to an orthogonal frame of reference aligned with the relative free-stream direction, resulting from the composition of the wind-tunnel free-stream and the relative rotational velocity. For convenience, the origin is placed at the leading edge of the airfoil. All the components of the velocity vector and the static pressure and density are required to be known along the contour.

\section{Uncertainty analysis}

\subsection{PIV measurement uncertainties}

Measurement uncertainties on the PIV velocity data contain random and bias components. The first ones are primarily caused by cross-correlation uncertainty, turbulent fluctuations and phase unsteadiness caused by jittering of timing systems. Due to statistical convergence, the effect of these components scales with $1 / \sqrt{ } N$ (with $N$ from 150 to 250 in the present analysis). For the cross-correlation uncertainty, a typical value of 0.1 pixel standard error is expected for a window size of $16 \times 16$ pixels (Westerweel 1993). On the other hand, the velocity fluctuations effect on the mean value is based on a maximum fluctuation level of $4 \%$ (stationary frame), highest value reported in the wake region of the analyzed fields. The minimum fluctuations observed on the field are of the order of $0.7 \%$, encountered at half a propeller diameter upstream the blade, which is substantially higher than the free-stream turbulence value of the LST wind-tunnel of less than $0.1 \%$. The overall uncertainty on the mean velocity due to random components is assessed at $0.1 \%$ in the steady regions and $0.4 \%$ of the free-stream velocity in the turbulent ones. The most relevant sources of systematic uncertainties in the PIV measurements are considered to be lack of spatial resolution, particle tracers slip, aero-optical aberrations, and peak locking.

The uncertainty due to particle tracer slip (Schrijer and Scarano 2007) has been found in the present investigation to be of relatively lower effect than in what was encountered in Ragni et al. 2009, although the blade chord is $1 / 5$ th of the NACA0012, primarily due to the relatively lower acceleration regions combined with the lower relaxation time of the SAFEX fog, estimated to be of the order of $1 \mu \mathrm{s}$. Of the same order of magnitude has been found, the velocity error caused by aero-optical aberrations, depending on the refractive index change in the field, hence on the density gradients, proportional to the (Elsinga et al. 2005) optical displacement field by the light ray deflection $\vec{\xi}$ as in Table 3. Statistical analysis of the smoothness of the resulting velocity histograms (Raffel et al. 2007) allowed assessing the peak-locking velocity error, depending on the particle image $d_{\tau}$, on the magnification $M$, on the separation time $\Delta t$, and on the particle displacement $\Delta_{p i x}$, to less than $0.15 \mathrm{~m} / \mathrm{s}$. The uncertainty related to spatial resolution is the most important, and it is determined by the ratio of the interrogation window size $w s$ to the spatial wavelength $\lambda$ of the flow feature under investigation (Schrijer and Scarano 2008). It hence depends strongly on the location in the flow, and for the present investigation, it is especially important in the wake region. The most relevant causes and 
Table 3 Summary of measurement uncertainty contributions for the velocity mean values

\begin{tabular}{|c|c|c|c|c|}
\hline & Uncertainties & Error estimator & Typical value & $\begin{array}{l}\text { Relative velocity } \\
\text { uncertainty }(\%)\end{array}$ \\
\hline Random components & $\begin{array}{l}\text { Fluctuations on the average } \\
\text { (cross-corr. and stat. convergence) }\end{array}$ & {$\left[\frac{\sigma_{\text {meas }}=f\left(\varepsilon, \sigma_{u}\right)}{\sqrt{N}}\right]$} & $\begin{array}{l}\varepsilon=0.1 \text { pixel } \\
\sigma_{u}=4 \%\end{array}$ & 0.35 \\
\hline \multirow[t]{4}{*}{ Systematic components } & Spatial resolution & {$[w s / \lambda]$} & $\lambda \sim 2 \mathrm{~mm}$ (wake) & $\leq 15$ \\
\hline & Peak locking & {$\left[\frac{d_{\tau}}{\Delta_{p i x} M \Delta t}\right]$} & $<0.03$ pixels & $<0.2$ \\
\hline & Aero-optical aberration & {$[(\vec{V} \cdot \nabla) \vec{\xi}]$} & & $\leq 2$ \\
\hline & Particle slip & {$[\tau \vec{V}(\nabla \vec{V})]$} & $\tau=1 \mu \mathrm{s}$ & $\leq 1.5$ \\
\hline
\end{tabular}

their estimated effects on the present PIV velocity fields are summarized in the following Table 3.

\subsection{Pressure and integral loads uncertainty}

Since isentropic relations can be applied to retrieve pressure and pressure coefficients in most of the domain, once the moving frame (steady in respect to the blade) is used, it is possible to derive by linear propagation analysis a direct relation between the error on velocity $\Delta V_{R}$ and the pressure coefficient $\Delta C_{p}$ :

$$
\begin{aligned}
C_{p} & =\frac{P-P_{\infty}}{\frac{1}{2} P_{\infty} \gamma M_{R \infty}^{2}}, \\
\Delta C_{p} & =-2 \frac{V_{R}^{2}}{V_{R \infty}^{2}}\left[1+\frac{(\gamma-1)}{2} M_{R \infty}^{2}\left(1-\frac{V_{R}^{2}}{V_{R \infty}^{2}}\right)\right]^{1 /(\gamma-1)} \frac{\Delta V_{R}}{V_{R}} \\
& =\kappa \frac{\Delta V_{R}}{V_{R}}=\eta \frac{\Delta V}{V}
\end{aligned}
$$

It is interesting to note that the square brackets contribution represents the density ratio $\rho / \rho_{\infty}$. The overall uncertainty multiplication parameter, dependent on the flow quantities, is indicated as the $\kappa$ or $\eta$ factor. The quantities state the amplification of the uncertainty from velocity to $C_{p}$, and they can be expressed both on the stationary and on moving frame. Since the PIV uncertainty is expressed in the stationary frame, $\eta$ is a better estimator for the uncertainty amplification; as an example, in the present investigation, with a relative increase of $V_{R} / V_{R \infty}$ of $1.4, \kappa=3.2$ and $\eta=1.12$; hence, 0.015 is the typical uncertainty on $C_{p}$.

The pressure variation in the vortical regions is obtained from integration of the momentum equation, by a secondorder Poisson algorithm, with isentropic boundary conditions, keeping the uncertainty on $C_{p}$ of the same order of the isentropic formulation. The uncertainty on the single values of the drag and lift coefficients has been based on the standard deviations of the different values found from the different contours. Results are shown in Table 4 together with the average values.

\section{Experimental results}

\subsection{Surface pressure evaluation}

To investigate in more detail, the pressure coefficient on the surface, the isentropic formulation together with the integration of the momentum equation has been used to compute the pressure fields from PIV. Subsequently, pressure profiles have been extracted along wall-normal lines and compared with data obtained from the CFD computation. Results of the PIV experiments are presented here for a revolution frequency of $350 \mathrm{~Hz}(21,000 \mathrm{rpm})$, at relative Mach number $M=0.60$.

In order to obtain the velocity (and pressure) distribution up to the surface of the blade on the suction side, only the camera with the most favorable viewing direction towards the blade surface has been used after being corrected for perspective effects. Sets of 150 images have been correlated by ensemble average of velocity vectors with window size of $4 \times 4$ pixels (about five vectors per mm with $0 \%$ overlap). Figure 6 shows a comparison between PIV and CFD of the relative velocity fields with the blade pitch angle at $15^{\circ}$. It has to be noted that the angle of attack of the incoming flow is much lower than the pitch angle, $\beta$ being about $0.6^{\circ}$, due to the composition of the wind-tunnel velocity $(48 \mathrm{~m} / \mathrm{s})$ with the rotational blade velocity $(194.5 \mathrm{~m} / \mathrm{s})$. The main features of the flow around the nonsymmetrical airfoil are comparable in the two snapshots; however, the stagnation point next to the leading edge and the decrease in velocity in the trailing edge are captured by CFD, while they are obscured by shadows in the PIV experiment. The suction side on the top and the pressure side on the bottom show comparable velocity contours in the two fields, with a maximum velocity of about 1.3 times the relative free stream $(=1.3 \times 200.6 \mathrm{~m} / \mathrm{s})$. With a Reynolds number of about 310,000 , the boundary layer is estimated to be less than $0.8 \mathrm{~mm}$, under the resolution capabilities of the PIV setup, not able to capture the velocity up to the blade surface. 
Table 4 Values and deviations of the aerodynamic coefficients

\begin{tabular}{lllll}
\hline a) Stationary frame formulation, $\mu \pm \sigma$ & & & \\
\hline$f(\mathrm{~Hz})$ & PIV & 350 & CFD & 350 \\
\cline { 2 - 5 } & 330 & $0.354 \pm 0.019$ & 330 & $0.316 \pm 0.002$ \\
\hline$\left|C_{y}\right|$ & $0.260 \pm 0.004$ & $0.076 \pm 0.006$ & $0.250 \pm 0.001$ & $0.091 \pm 0.003$ \\
$\left|C_{x}\right|$ & $0.080 \pm 0.001$ & $0.362 \pm 0.017$ & $0.096 \pm 0.002$ & $0.323 \pm 0.002$ \\
$\left|C_{l}\right|$ & $0.275 \pm 0.005$ & $0.008 \pm 0.011$ & $0.263 \pm 0.001$ & $0.014 \pm 0.003$ \\
$\left|C_{d}\right|$ & $0.013 \pm 0.001$ & & $0.024 \pm 0.002$ &
\end{tabular}

b) PIV Stationary frame formulation, $\mu \pm \sigma$

\begin{tabular}{lllll}
\hline$f(\mathrm{~Hz})$ & 330 & & & \\
\cline { 2 - 5 }$\alpha(\mathrm{deg})$ & -10 & -5 & 5 & 10 \\
\hline$\left|C_{y}\right|$ & $0.269 \pm 0.010$ & $0.262 \pm 0.010$ & $0.263 \pm 0.004$ & $0.277 \pm 0.006$ \\
$\left|C_{x}\right|$ & $0.100 \pm 0.003$ & $0.094 \pm 0.006$ & $0.104 \pm 0.005$ & $0.105 \pm 0.005$ \\
$\left|C_{l}\right|$ & $0.287 \pm 0.010$ & $0.277 \pm 0.011$ & $0.283 \pm 0.002$ & $0.294 \pm 0.007$ \\
$\left|C_{d}\right|$ & $0.029 \pm 0.001$ & $0.025 \pm 0.003$ & $0.032 \pm 0.003$ & $0.029 \pm 0.003$
\end{tabular}

PIV Moving-frame formulation, $\mu \pm \sigma$

\begin{tabular}{lllll}
\hline$f(\mathrm{~Hz})$ & 330 & & & \\
\cline { 2 - 5 }$\alpha(\mathrm{deg})$ & -10 & -5 & 5 & 10 \\
\hline$\left|C_{l}\right|$ & $0.243 \pm 0.009$ & $0.270 \pm 0.012$ & $0.330 \pm 0.009$ & $0.340 \pm 0.015$ \\
\hline
\end{tabular}

Fig. 6 Left PIV relative velocity field at $3 / 4$ propeller radius, revolution frequency $350 \mathrm{~Hz}$; right CFD relative velocity field at the same regime
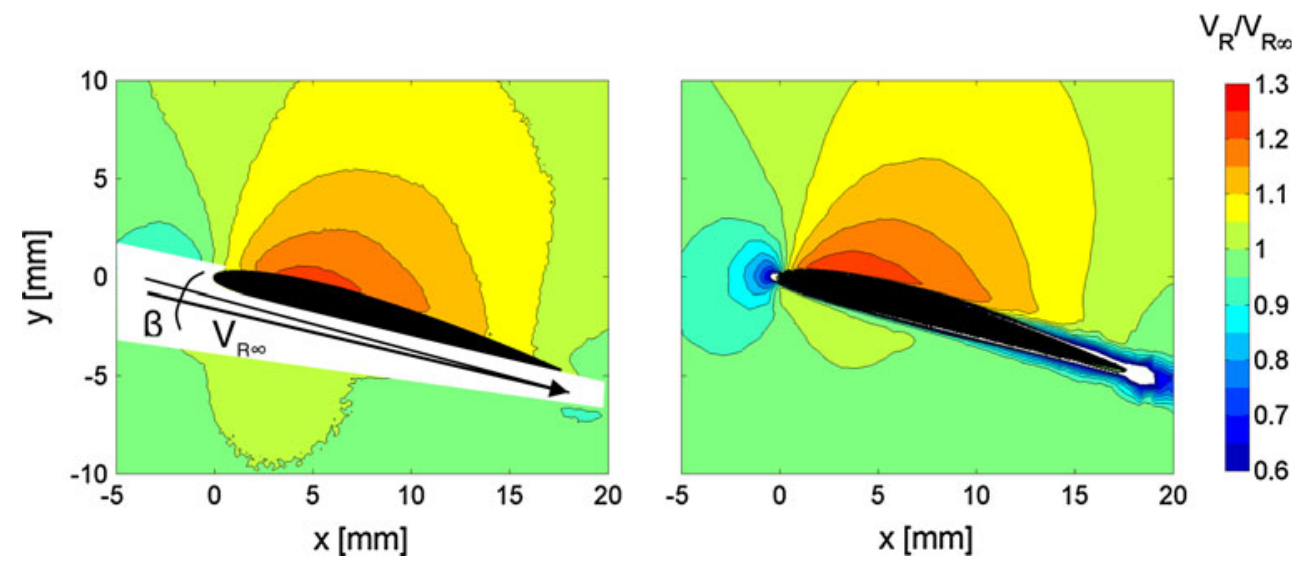

In Fig. 7, the pressure coefficient is derived from integration of the velocity fields from PIV with isentropic boundary conditions and compared with the pressure from the CFD simulation; the contours maintain the previously mentioned overall similarities, with a significant drop of pressure in the suction side, not balanced by the one on the lower side.

The two fields have been used to compare the pressure on the suction side of the blade along lines normal to the blade surface, and subsequently, the results were linearly extrapolated to obtain the pressure at the surface. The extracted pressure profiles are plotted in Fig. 8, with the
PIV data indicated by blue triangles, whereas the CFD measurements are given by the green circles. The corresponding surface pressure distributions are plotted in Fig. 9. The PIV-based pressure data in Figs. 8 and 9 are found in good agreement with the CFD computation; albeit that the PIV measurements become unreliable close to the leading edge due to reflections and edge effects (high degrees of flow acceleration). The raw PIV-based surface pressure distributions provided in Fig. 9 (blue symbols) have been taken at $0.25 \mathrm{~mm}$ distance from the wall ( $1 \%$ chord). Relative to the CFD data, the linear extrapolation of the PIV pressure data (red symbols) 
Fig. 7 Left PIV pressure coefficient velocity field at $3 / 4$ propeller radius, revolution frequency $350 \mathrm{~Hz}$; Right CFD relative velocity field at the same regime
Fig. 8 Pressure coefficients along lines normal to the airfoil profile, in blue PIV, in green $\mathrm{CFD}$, in red linear extrapolation up to the surface
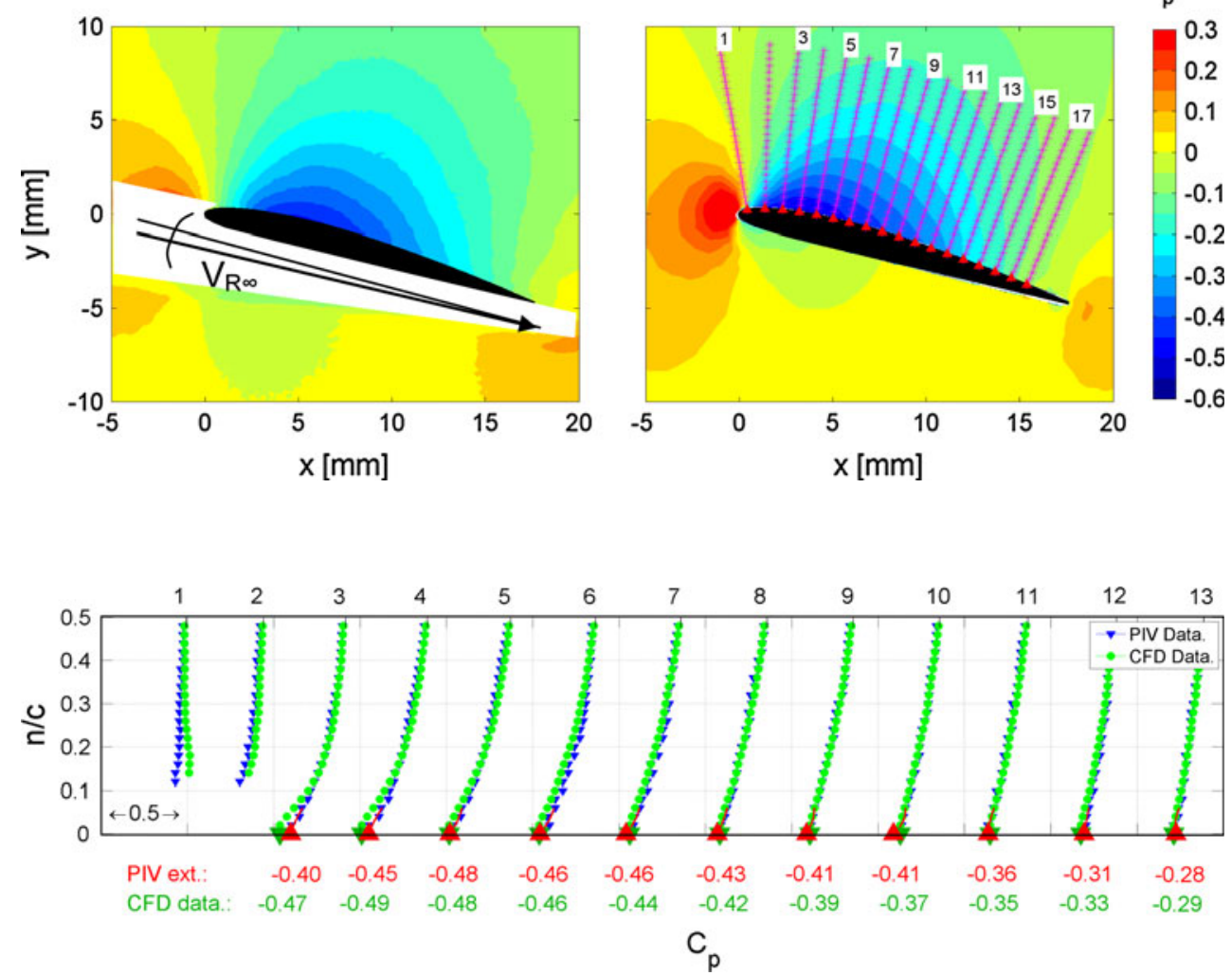

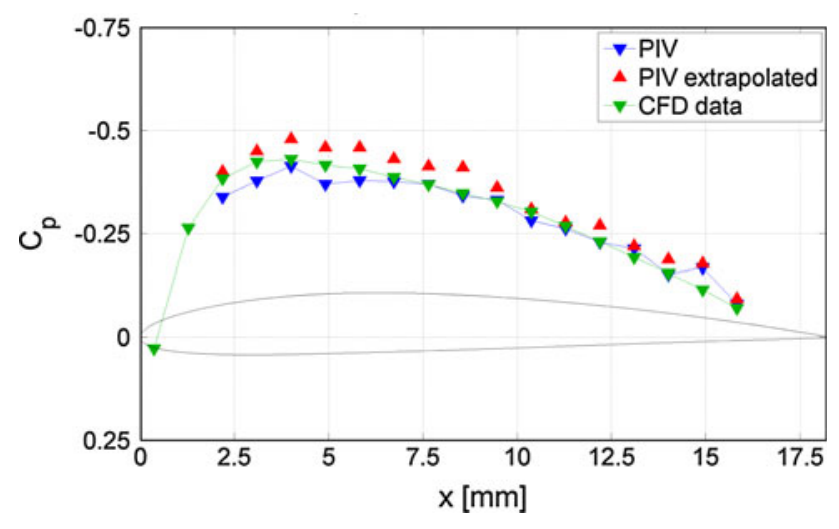

Fig. 9 Surface pressure coefficient on the suction side as computed from PIV and CFD

shows a significant improvement only next to the leading edge.

\subsection{Integral force determination}

In order to compute the integral lift force by means of the contour approach, a field of view encompassing the airfoil is needed. In the present investigation, as can be seen from the experimental setup of Fig. 2, the profile is creating a shadow region with a height of about $4 \mathrm{~mm}$ upstream of the airfoil. Due to the regularity of the flow in the stagnation region and the expected behavior of the potential velocity field, a second-order interpolation of the data is carried out through the missing points of the velocity fields to fill in this shadow region at some distance from the airfoil. The distribution of the magnitude of (relative) velocity is shown on Figs. 10 and 11 left, in which the interpolated side is on the left. The velocity fields show a similar flow pattern as in the previous field, with a larger extent of the suction region. The results for the relative velocity and derived pressure fields obtained with the two different reference-frame formulations are compared in Figs. 10 and 11: negligible differences are detectable between the moving and the stationary formulation for the pressure determination, notably in the wake region, where the stationary formulation appears to yield slightly smoother results.

The previous fields have been further exploited to compute lift and drag coefficients by the momentum contour approach. In propeller aerodynamics, the lift and drag forces on the single blade profiles can be projected into the propeller frame of reference $x-y-z$ as horizontal and tangential components, building up the propeller resistance $F_{x}$, thrust $F_{y}$. A variable number of rectangular contours with ratio width/height $4 / 1$ spaced by $1 \mathrm{~mm}$ between 15 and 20 have been chosen to determine the values and the variability of the lift and drag coefficients. Given the low 

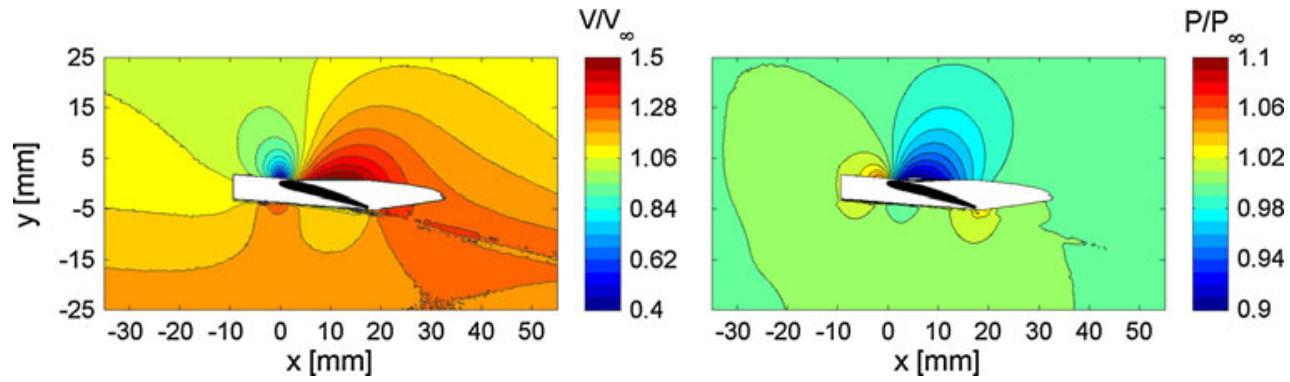

Fig. 10 Velocity magnitude and Poisson evaluated pressure from stationary frame formulation with time derivatives included

Fig. 11 Relative velocity magnitude and Poisson evaluated pressure from moving-frame formulation
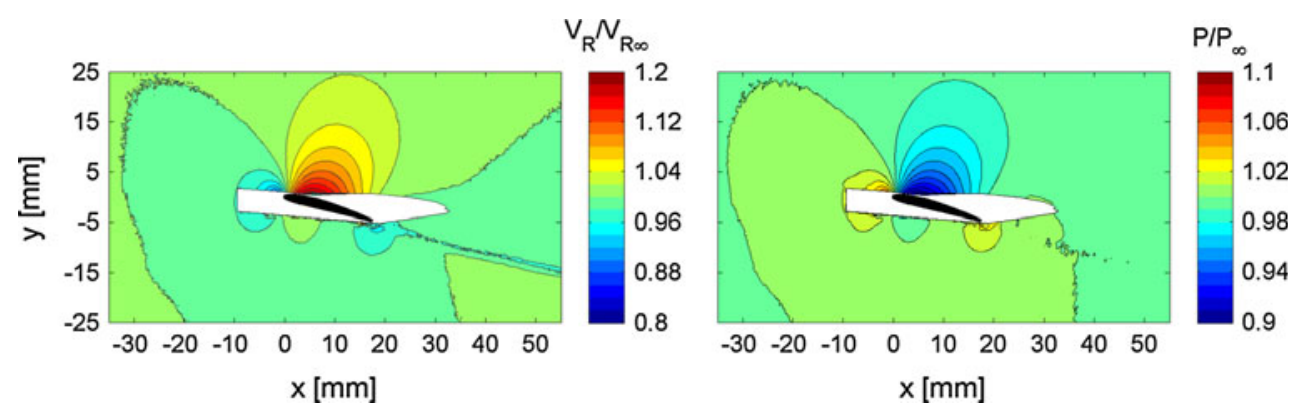

scaling factor and the window size adopted, the contour approach for the drag coefficient determination suffers from a severe lack of resolution, which is typically less affecting the lift computation (see e.g. van Oudheusden et al. 2006; Ragni et al. 2009). In fact the cases where the extent of the wake is not clearly defined or not captured by the field of view, the uncertainties in the drag coefficient computation become much larger. The following tables summarize the values obtained by integration of the PIV fields compared with the ones computed by the CFD simulation for both the blade frame (lift and drag coefficients) and the propeller frame (thrust and resistance).

Table 4a compares the values obtained from the PIV computation to the ones obtained from CFD for the two symmetrical cases at different revolution frequencies. A remarkable difference in the order of $10 \%$ for the lift coefficient has been found between the PIV experiments and the CFD numerical simulation, which can be justified by the non-exact reproduction of the $3 \mathrm{D}$ blade shape and by small differences in the free-stream conditions. The stationary frame formulation has been found to give comparable results to the moving one in Table $4 \mathrm{~b}$; however, a consistent difference between the two derivations is present in yawing conditions, which was not seen in no-yawing conditions. In this respect, it has to be noted that the application of the contour approach in the stationary frame requires the integral in the internal points of the time derivative $\rho \bar{V}$ as in Eq. 6. The presents of reflection reduces the availability of some points in the pressure side of the blade, determining the difference with the moving frame, more robust with the absence of the time derivative.
The uncertainties in the moving-frame formulation are too large to define the relatively small drag coefficient with acceptable accuracy. Small differences can be appreciated in both drag and lift coefficients at different yawing angles with a small increase of the quantities with yawing, primarily due to the relatively small change in the out of plane components, found to be changing in a range from $\pm 10 \mathrm{~m} / \mathrm{s}$.

\section{Conclusions}

An experimental campaign by means of PIV has been carried out on a scaled model of a propeller aircraft running at transonic conditions, with the intent to investigate the pressure distribution around the blade as well as the local aerodynamic loads at different yawing angles of the propeller. Phase-locked measurement proved to phase-resolve the periodical flow of the blade revolution, allowing using either the moving frame of reference, blade based, or the stationary frame of reference, laboratory based. The technique demonstrated that the local velocity information on a blade section can be coupled with the global load information determined as force reaction balance on the flow itself. Integration of the Navier-Stokes equation in the compressible regime was used for a direct comparison of the pressure field coefficients derived from the measured velocity data, with the ones of a numerical RANS-periodical steady computation of the propeller model at two experimental revolution frequencies $(330,350 \mathrm{~Hz})$. The pressure fields have been reconstructed by integration of 
the momentum equation by a Poisson reconstruction algorithm; subsequently, the pressure coefficient has been extracted from the PIV data along lines normal to the profile surface and compared with the ones derived from CFD computations. A reasonable agreement has been found between the two methods, with a maximum mismatch on the order of $10 \%$, in the entire measurable PIV region. The pressure fields together with the velocity data have been integrated with a contour approach in order to give information about the aerodynamic force components, mainly lift and drag. The PIV-based lift coefficient was found to be comparable to the CFD-based one, within the same order of magnitude found in the pressure coefficient analysis, while the drag component remains in the limit of the PIV uncertainty due to the relatively low real-angle of attack and to the relatively small wake imbalance, showing differences of the order of $20 \%$. No important differences have been found in the local load in yawing, by both the moving and the stationary frame formulation, due to the relatively small change in the out-of-plane velocity component.

Open Access This article is distributed under the terms of the Creative Commons Attribution Noncommercial License which permits any noncommercial use, distribution, and reproduction in any medium, provided the original author(s) and source are credited.

\section{References}

Anderson JD (1991) Fundamentals of aerodynamics, 2nd edn. McGraw Hill Publishers, NY

Bousquet JM, Gardarein P (2003) Improvements on computations of high speed propeller unsteady aerodynamics. Aerospace Sci Technol 7(6):465-472

Boyle FJ, Flaherty MPO, Eaton JA (1999) Comparison of PIV measurements with CFD predictions for a high speed propeller, 35th AIAA/ASME/SAE/ASEE joint propulsion conference and exhibit, Los Angeles, California, AIAA 99-2387
Calgano G, Di Felice F, Felli M, Pereira F (2005) A stereo-PIV Investigation of a propeller's wake behind a ship model in a large free-surface tunnel. Marine Tech Soc J 39(2):99-102

Elsinga GE, van Oudheusden BW, Scarano F (2005) Evaluation of aero-optical distortion effects in PIV. Exp Fluids 39(2):245-256

Favier D, Maresca C, Ettaouil A (1989) Numerical investigation of isolated propeller wakes in axial flight. J Aircraft 26:837-846

Raffel M, Willer C, Wereley S, Komphenhans J (2007) Particle image velocimetry, a practical guide, 2nd edn. Springer, Berlin

Ragni D, Ahok A, van Oudheusden BW, Scarano F (2009) Surface pressure and aerodynamic loads determination of a transonic airfoil based on particle image velocimetry. Meas Sci Tech 20(7):1-14

Ramasamy M, Leishman JG (2006) Benchmarking PIV with LDV for rotor wake vortex flows, AIAA 2006-3479, 24th applied aerodynamics conference 5-8 June 2006, San Francisco, California

Roosenboom EWM, Stürmer A, Schröder A (2010) Advanced experimental and numerical validation and analysis of propeller slipstream flows. AIAA 47(1):284-291

Scarano F, Riethmuller ML (2000) Advances in iterative multi-grid PIV image processing. Exp Fluids 29(1):51-60

Schrijer FFJ, Scarano F (2007) Particle slip compensation in steady compressible flows, 7th international symposium on particle image velocimetry. Rome, Italy

Schrijer FFJ, Scarano F (2008) Effect of predictor-corrector filtering on the stability and spatial resolution of iterative PIV interrogation. Exp Fluids 45(5):927-941

Trefethen L (2000) Spectral methods in Matlab. SIAM, Philadelphia

Unal MF, Lin JC, Rockwell D (1997) Force prediction by PIV imaging: a momentum-based approach. J Fluids Struct 11:965-971

van Oudheusden BW (2008) Principles and application of velocimetry-based planar pressure imaging in compressible flows with shocks. Exp Fluids 45(4):657-674

van Oudheusden BW, Scarano F, Casimiri EWM (2006) Nonintrusive load characterization of an airfoil using PIV. Exp Fluids 40(6):988-992

van Oudheusden BW, Scarano F, Roosenboom EWM, Casimiri EWF, Souverein LJ (2007) Evaluation of integral forces and pressure fields from planar velocimetry data for incompressible and compressible flows. Exp Fluids 43(2-3):153-162

Westerweel J (1993) Digital particle image velocimetry. Delft University Press, Delft 